\title{
Could hepatic ablation promote development of colon cancer hepatic metastases?
}

\author{
Crystal Kyaw ${ }^{1}$, Joyce Wong ${ }^{2}$ \\ ${ }^{1}$ Department of Surgery, Lenox Hill Hospital at Northwell Health, New York, NY 10075, USA; ${ }^{2}$ Department of Surgery, Kaiser Permanente Mid- \\ Atlantic Medical Group, Washington, DC 20002, USA \\ Correspondence to: Joyce Wong, MD FACS. Capitol Hill Medical Center, 700 2nd St. NE, Washington, DC 20002, USA. \\ Email: joyce.wong02@gmail.com. \\ Provenance and Peer Review: This article was commissioned by the editorial office, Hepatobiliary Surgery and Nutrition. The article did not undergo \\ external peer review. \\ Comment on: Jones EL, Halpern AL, Carmichael H, et al. Hepatic Ablation Promotes Colon Cancer Metastases in an Immunocompetent Murine \\ Model. Ann Surg 2019;270:675-80.
}

Submitted Oct 22, 2019. Accepted for publication Nov 21, 2019.

doi: $10.21037 /$ hbsn.2019.11.05

View this article at: http://dx.doi.org/10.21037/hbsn.2019.11.05

\section{Introduction}

Colon cancer is currently the third most common cancer and cause of cancer-related deaths in the United States. Approximately $35-55 \%$ of patients with colorectal cancer present with metastatic disease; of these, $10-20 \%$ of patients have resectable disease, with the liver being the most common site of metastasis (1). Surgical resection is considered the gold standard curative option for metastatic disease, with overall 5 -yr survival of 35-55\% after successful resection (1). Many patients with surgically unresectable disease, however, are candidates for microwave (MW) or radiofrequency ablation (RFA) of hepatic metastases or other liver-directed therapies. While ablation can often be performed minimally invasively or percutaneously and is generally well tolerated with effective local tumor control, it is not without limitations.

Three main limitations of RFA are often cited. First, this technique is limited by the volume of tissue to be ablated. While individual protocols may vary, $4-5 \mathrm{~cm}$ maximum diameter is generally accepted as the upper limit of what can be ablated without significant risk of hepatic abscess or injury; this typically would account for treatment of a tumor $3 \mathrm{~cm}$ or smaller (2). Second, this technique can be technically infeasible due to tumor location or local characteristics. Attempting to ablate a lesion adjacent to the colon, gallbladder or bile ducts, for instance, can put these organs at risk for thermal injury. Various techniques to minimize this injury have been developed, including creation of artificial ascites to assist RFA by separating tissue planes, and biliary cooling via a naso-biliary catheter to lessen the thermal impact on biliary structures. Third, the heat-sink effect seen most prominently with lesions near vessels can limit its efficacy. The thermal impact of the ablation is dissipated by blood flow away from the lesion, noticed most when the ablative tip of the probe is within close proximity of a major hepatic vein. The use of a Pringle maneuver has been noted to minimize this heat sink effect, though this then requires operative intervention.

Despite these limitations, ablative techniques are used increasingly more often for their benefits as a targeted, often minimally invasive, alternative of addressing hepatic lesions. In one series evaluating a percutaneous approach for 61 lesions, complete ablation was achieved in $86.9 \%$; recurrence was predicted by the presence of multiple metastases, ablation margins $<5 \mathrm{~mm}$, and non-colorectal metastases (3). Up to $50 \%$ of patients, however, ultimately develop recurrent metastatic disease after initial ablative or surgical treatment (4). Prognostic factors include multiple lesions and elevated CEA and CA19-9 levels. Other factors that have also been identified include number of lesions, size, and bilobar distribution (5-8).

\section{Synopsis of paper}

Jones et al. present an intriguing murine model of thermal 
hepatic injury in the setting of hematogenous instillation of colon adenocarcinoma cells to promote metastases formation (9). They evaluate the rate of formation of metastatic disease following various modalities of ablative therapy, including RFA, MW, and direct cautery. While RFA has been widely used to treat small or unresectable liver metastases, $\mathrm{MW}$ is increasingly being used. Both of these modalities increase tissue temperature to induce protein coagulation and cell death. When compared to surgical resection, however, both these therapies have demonstrated increased local recurrence rates and decreased survival, although these are typically not matched cohorts and represent a heterogeneous group. The authors hypothesize that direct cautery may be associated with a lower metastatic recurrence rate due to its pattern of more localized thermal spread than that seen in either MW or RFA, while still allowing adequate heating of the tissue.

The study was conducted with four groups: RFA, MW, cautery, sham. Tumor cells were introduced via splenic injection just prior to application of either RFA, MW, cautery energy, or simple puncture injury in the case of the sham group. Thermography was performed with an infrared camera to monitor tissue temperatures, and the mice were sacrificed POD7 to measure tumor volume. Tissue temperature was significantly higher for cautery than either MW of RFA, but the rate of temperature decline was significantly faster, and final tissue temperature was lower in the cautery group; the mean tumor volume was significantly lower in the direct cautery group as compared to $\mathrm{MW}$ or RFA, which were similar to each other. Interestingly, no metastases were seen in the sham mice receiving non-thermal probe injury.

This study seems to suggest that utilization of RFA or MW creates a milieu in the hepatic parenchyma that allows for tumor cells to adhere and populate. This, perhaps, reinforces the concept that circulating tumor cells (CTCs) may be present during surgical treatment; CTCs have been promoted as one theory to explain metastasis formation from colon cancer despite surgical resection. This finding could have major implications for the management of metastatic colorectal cancer. This is the first study to use thermal imaging to compare direct cautery to $M W$ and RFA using an in vivo animal model. Ultimately, the authors conclude that the rapid heating and destruction of liver tissue results in less tumor recurrence. Thus, by reducing the area of subacute injury that results from MW or RFA, for example, the incidence of metastatic recurrence may be limited (9).

\section{Discussion}

This study, provocative in nature, raises the question about clinical application of current ablative modalities; there would be grave concern if RFA or MW inadvertently alters the hepatic parenchyma to make it favorable for metastatic tumors to populate. One question raised by a discussant focused on the lack of visible lesions, which would, in clinical terms, obviate any ablation application. Additionally, most patients typically undergo systemic treatment prior to surgical resection or liver-directed therapy. This would again relate to the concept of CTCs creating metastatic disease. Cohen et al. outlined the poor prognosis of patients with metastatic colorectal carcinoma and demonstrated the association of poor overall and progression-free survival with higher levels of CTCs. These levels could persist despite administration of months of systemic therapy (10). Creation of a niche or microenvironment for CTCs to implant upon, however, with the utilization of ablative techniques is a novel concept.

Initial concern regarding local recurrence following RFA or MW centered on the zones of coagulation, particularly for RFA. Three zones typically are created: a center charred zone, coagulation zone, and congestion zone (11). These zones are dependent upon tissue heterogeneity since electric and thermal conductivity varies by tissue type; these zones may also not be symmetric. Longer ablations with microwave seemed to improve upon the shape and zone of coagulation. Because the zone of coagulation and congestion are not necessarily associated with true cell death, it has been hypothesized that these zones contribute to local tumor recurrence. Ensuring one $\mathrm{cm}$ margins around the lesion has been associated with lower tumor recurrence (12).

Several studies have evaluated the changes that occur at the periphery of the necrotic zone, and have found that a strong immunologic response occurs. Neutrophils, activated myofibroblasts and macrophages infiltrate into the surrounding zone, and there is a substantial increase in various factors such as hepatocyte growth factor and interleukin-6 (13). Another group also demonstrated in vivo that the pro-inflammatory response of the hepatic parenchyma around the ablation promoted hepatic metastases, particularly because of increased expression of vascular endothelial growth factor and fibroblast growth factor-2 (14). This pro-inflammatory milieu has been demonstrated even in non-thermal ablative technologies, such as irreversible electroporation (IRE); in particular with 
IRE, where vasculature is preserved because of the nature of ablation, there seems to be a stronger upregulation of inflammatory cytokines (15). It is unclear whether this will translate to a higher recurrence rate clinically.

These studies raise a startling concept: perhaps by ablating tumors, we are creating a microenvironment which is a great substrate for metastasic tumors to grow. Outside of in vivo applications, however, human patients and clinical application are much more complex. Patients typically undergo multi-modality therapy, which includes systemic therapy, surgery, and other liver directed therapies. These include chemoembolization, radioembolization and even hepatic artery infusion pumps. These modalities will also change the hepatic parenchyma and its response to subsequent ablation. In situations of a pre-treated liver, it is difficult to draw the same conclusion that the peritumoral ablation site increases the pro-tumorigenic microenvironment.

\section{Conclusions}

Surgical resection remains the mainstay of treatment for colorectal liver metastases. However, ablation techniques have been increasing in frequency but are affiliated with a significant risk of local recurrence. Based on this study by Jones et al., it is possible that the pro-inflammatory effect of ablation creates a niche for metastatic cells to implant and grow. However, in the context of patients receiving systemic and potentially other liver-directed therapies, it is unclear if this, as yet unproven risk, outweighs the benefit of ablation. Further investigation is needed to understand the role of ablation in the context of treatment of colorectal liver metastases.

\section{Acknowledgments}

Funding: None.

\section{Footnote}

Conflicts of Interest: The authors have no conflicts of interest to declare.

Ethical Statement: The authors are accountable for all aspects of the work in ensuring that questions related to the accuracy or integrity of any part of the work are appropriately investigated and resolved.
Open Access Statement: This is an Open Access article distributed in accordance with the Creative Commons Attribution-NonCommercial-NoDerivs 4.0 International License (CC BY-NC-ND 4.0), which permits the noncommercial replication and distribution of the article with the strict proviso that no changes or edits are made and the original work is properly cited (including links to both the formal publication through the relevant DOI and the license). See: https://creativecommons.org/licenses/by-ncnd/4.0/.

\section{References}

1. Al Bandar MH, Kim NK. Current status and future perspectives on treatment of liver metastasis in colorectal cancer (Review). Oncol Rep 2017;37:2553-64.

2. Rhim $\mathrm{H}$ and Lim H. Radiofrequency Ablation of Hepatocellular Carcinoma: Pros and Cons. Gut Liver 2010;4:S113-8.

3. Sparchez Z, Mocan T, Hajjar NA, et al. Percutaneous ultrasound guided radiofrequency and microwave ablation in the treatment of hepatic metastases. A monocentric initial experience. Med Ultrason 2019;21:217-24.

4. Kim WJ, Lim TW, Kang SH, et al. Development and validation of novel scoring system for the prediction of disease recurrence following resection of colorectal liver metastasis. Asian J Surg 2020;43:438-46.

5. Chan KM, Wu TH, Cheng CH, et al. Prognostic significance of the number of tumors and aggressive surgical approach in colorectal cancer hepatic metastasis. World J Surg Oncol 2014;12:155.

6. Homayounfar K, Bleckmann A, Conradi LC, et al. Bilobar spreading of colorectal liver metastases does not significantly affect survival after $\mathrm{R} 0$ resection in the era of interdisciplinary multimodal treatment. Int J Colorectal Dis 2012;27:1359-67.

7. Fong Y, Fortner J, Sun RL, et al. Clinical score for predicting recurrence after hepatic resection for metastatic colorectal cancer: analysis of 1001 consecutive cases. Ann Surg 1999;230:309-18.

8. Konopke R, Kersting S, Distler M, et al. Prognostic factors and evaluation of a clinical score for predicting survival after resection of colorectal liver metastases. Liver Int 2009;29:89-102.

9. Jones EL, Halpern AL, Carmichael H, et al. Hepatic 
Ablation Promotes Colon Cancer Metastases in an Immunocompetent Murine Model. Ann Surg 2019;270:675-80.

10. Cohen SJ, Punt CJA, Iannotti N, et al. Relationship of Circulating Tumor Cells to Tumor Response, Progression-Free Survival, and Overall Survival in Patients With Metastatic Colorectal Cancer. J Clin Oncol 2008;26:3213-21.

11. Zhou W, Liang M, Pan H, et al. Comparison of Ablation Zones among Different Tissues Using 2450-MHz CooledShaft Microwave Antenna: Results in Ex Vivo Porcine Models. PLoS One 2013;8:e71873.

12. Mulier S, Ni Y, Jamart J, et al. Local Recurrence After Hepatic Radiofrequency Coagulation. Multivariate Meta-

Cite this article as: Kyaw C, Wong J. Could hepatic ablation promote development of colon cancer hepatic metastases? HepatoBiliary Surg Nutr 2020;9(3):364-367. doi: 10.21037/ hbsn.2019.11.05
Analysis and Review of Contributing Factors. Ann Surg 2005;242:158-71.

13. Rozenblum N, Zeira E, Bulvik B, et al. Radiofrequency Ablation: Inflammatory changes in the Periablative Zone Can Induce Global Organ Effects, including Liver Regeneration. Radiology 2015;276:416-25.

14. Nikfarjam M, Muralidharan V, Christophi C. Altered growth patterns of colorectal liver metastases after thermal ablation. Surgery 2006;139:73-81.

15. Bulvik BE, Rozenblum N, Gourevich S, et al. Irreversible Electroporation versus Radiofrequency Ablation: A Comparison of Local and Systemic Effects in a SmallAnimal Model. Radiology 2016;280:413-24. 